\title{
P-26
}

\section{Mesua Beccariana, a Source of Potential Cytotoxic Compounds}

\author{
Gwendoline Cheng Lian Ee ${ }^{1, *}$, Soek Sin Teh ${ }^{1}$, Siau Hui Mah ${ }^{1}$, Yang Mooi Lim², Mawardi Rahmani ${ }^{1}$ \\ ${ }^{I}$ Department of Chemistry, Faculty of Science, Universiti Putra Malaysia, 43400 UPM Serdang, Selangor, Malaysia; \\ ${ }^{2}$ Faculty of Medicine and Health Science, Universiti Tunku Abdul Rahman, 43000, Kajang, Selangor, Malaysia; \\ E-mail: gwen@science.upm.edu.my
}

Phytochemists nowadays have growing interests on medicinal plants which possess pharmacological activities. Therefore, an investigation on biologically active secondary metabolites from the stem bark of Mesua beccariana (Clusiaceae) was carried out. Two new compounds, a cyclodione which is mesuadione 1 and a coumarin, beccamarin 2 along with several other constituents were obtained from our ongoing research. These compounds were identified to be mesuarianone 3, mesuasinone 4, 2,5-dihydroxy1,3,4-trimethoxyanthraquinone 5 and 4-methoxy-1,3,5-trihydroxy anthraquinone 6 . The structures of these compounds were elucidated by extensive spectroscopic methods which include 1D and 2D-NMR, GC-MS, IR and UV techniques. Cytotoxic activities of all the isolated metabolites against a panel of human cancer cell lines including Raji (lymphoma), SNU-1 (stomach), LS-174T (colon), HeLa (cervical), SK-MEL-28 (skin), NCI-H23 (lung), IMR-32 (neuroblastoma), Hep-G2 (liver) and K562 (leukemia) were performed. All the secondary metabolites exhibited significant to weak cell proliferation inhibition against all the investigated cancer cells. The cytotoxic activities were evaluated using the MTT assay.

Keywords: Clusiaceae, Mesua beccariana, Cyclodione, Coumarin, Cytotoxic. 